\title{
Study of increased radiation when an x-ray tube is placed in a strong magnetic field
}

\author{
Zhifei Wen \\ Departments of Radiology and Physics, Stanford University, Stanford, California 94305 \\ Norbert J. Pelc \\ Departments of Radiology and Bioengineering, Stanford University, Stanford, California 94305 \\ Walter R. Nelson \\ Radiation Physics Group, Stanford Linear Accelerator Center, Stanford, California 94309 \\ Rebecca Fahrig \\ Department of Radiology, Stanford University, Stanford, California 94305
}

\begin{abstract}
When a fixed anode $\mathrm{x}$-ray tube is placed in a magnetic field $(B)$ that is parallel to the anode-cathode axis, the x-ray exposure increases with increasing $B$. It was hypothesized that the increase was caused by backscattered electrons which were constrained by $B$ and reaccelerated by the electric field onto the x-ray tube target. We performed computer simulations and physical experiments to study the behavior of the backscattered electrons in a magnetic field, and their effects on the radiation output, x-ray spectrum, and off-focal radiation. A Monte Carlo program (EGS4) was used to generate the combined energy and angular distribution of the backscattered electrons. The electron trajectories were traced and their landing locations back on the anode were calculated. Radiation emission from each point was modeled with published data (IPEM Report 78), and thus the exposure rate and $\mathrm{x}$-ray spectrum with the contribution of backscattered electrons could be predicted. The point spread function for a pencil beam of electrons was generated and then convolved with the density map of primary electrons incident on the anode as simulated with a finite element program (Opera-3d, Vector Fields, UK). The total spatial distribution of $\mathrm{x}$-ray emission could then be calculated. Simulations showed that for an X-ray tube working at $65 \mathrm{kV}$, about $54 \%$ of the electrons incident on the target were backscattered. In a magnetic field of $0.5 \mathrm{~T}$, although the exposure would be increased by $33 \%$, only a small fraction of the backscattered electrons landed within the focal spot area. The x-ray spectrum was slightly shifted to lower energies and the half value layer (HVL) was reduced by about 6\%. Measurements of the exposure rate, half value layer and focal spot distribution were acquired as functions of $B$. Good agreement was observed between experimental data and simulation results. The wide spatial distribution of secondary x-ray emission can degrade the MTF of the $\mathrm{x}$-ray system at low spatial frequencies for $B \leqslant 0.5 \mathrm{~T}$.
\end{abstract}

Key words: increased radiation, backscattered electrons, X-ray tube, magnetic field, XMR

\section{INTRODUCTION}

The combination of two powerful imaging modalities, magnetic resonance (MR) imaging and x-ray fluoroscopy, can potentially provide innovative image guidance for a variety of minimally invasive interventional procedures. In our hybrid system implementation, a fixed anode x-ray tube and a flat-panel detector (Revolution, GE Healthcare, Milwaukee, WI) are placed inside the magnet of an open-bore MR system (GE Signa SP, GE healthcare, Milwaukee, WI). ${ }^{1,2}$ After the $\mathrm{x}$-ray and MR systems were physically integrated, the image quality of each system was evaluated. ${ }^{3}$ We observed that the $\mathrm{x}$-ray tube output increased by more than $30 \%$ when the $\mathrm{x}$-ray source was placed in a $0.5 \mathrm{~T}$ magnetic field.

When high energy electrons strike the target in an x-ray tube, a substantial number of electrons are backscattered. It has been reported that backscattered electrons in an x-ray tube with a grounded cathode could increase the radiation output and change the $\mathrm{x}$-ray spectrum ${ }^{4}$ since the electric field drives backscattered electrons back to the anode. Similarly, we hypothesized that the output increase observed for our tube placed in the magnetic field was caused by the backscattered electrons. In a strong magnetic field that is aligned with the cathode-anode axis of the $\mathrm{x}$-ray tube, backscattered electrons are confined to helical motions about the magnetic field lines and therefore are more likely to be accelerated back onto the anode to produce secondary radiation. An external magnetic field will also, of course, have effects on the electron trajectories as the electrons are accelerated from the cathode to the anode. These effects have been investigated elsewhere. 5,6

In order to provide further evidence for the hypothesis of confined backscattered electrons, we performed computer simulations and careful measurements of an x-ray tube oper- 
ating in a magnetic field. Simulation results, in good agreement with experimentally measured data, support the hypothesis.

\section{METHODS}

We first explain the physical processes that electrons undergo in an x-ray tube, together with computer simulations that describe these processes. We then use computer simulations to generate observable quantities and measure those quantities experimentally. These quantities include the radiation exposure rate, $x$-ray spectrum, half value layer (HVL), and off-focal radiation. Finally, we predict how the amount and distribution of the increased radiation will affect the $\mathrm{x}$-ray system response.

\section{A. Physical processes of electrons in an x-ray tube 1. Electron beam optics}

In an x-ray tube, a cathode and an anode are placed inside a vacuum envelope, with an accelerating potential between them. The cathode emits electrons that are accelerated by the applied electric field. In our case, the intended target for the electrons is the focal spot area of tungsten on a static anode. $\mathrm{X}$ rays are produced by interactions between the high-energy electrons and tungsten atoms. To limit the focal spot size, a focusing cup around the cathode shapes the electric field so that electrons straying away from the tube axis are pushed back towards the center.

A finite-element-method program (OPERA-3d; Vector Fields, Kidlington, Oxford, UK) was used to simulate the electron trajectories in a geometry similar to that of our fixed anode x-ray tube. ${ }^{5,7}$ The electrostatic Poisson's equation for each finite element was solved numerically to obtain the electrostatic field in space, and electron trajectories were calculated by tracing their motions in the elements. The electron distribution on the focal spot was obtained by plotting the electron density map near the anode surface.

\section{Backscattered electrons}

The collisions between the primary electrons and the anode material cause a large number of the electrons to escape from the anode and re-enter the space between the cathode and anode with lower energies and in all directions. Under the influence of the electric field of the tube and in the absence of a magnetic field, these backscattered electrons first travel toward the cathode while being decelerated, reach zero velocity in the direction of the tube axis, and then are reaccelerated toward the anode until they hit it again with the same energies as they had when they escaped the anode. Depending on their energies and directions, some of the electrons land within the tungsten target area while others may not (Fig. 1). Those that hit the tungsten target are more likely to produce $\mathrm{x}$-ray radiation than those that hit copper. We predict the locations at which the backscattered electrons hit based on their backscattering energies and directions. From geometric symmetry, the backscattered electrons should be uniformly distributed in the azimuthal angle $(\phi)$, and there-

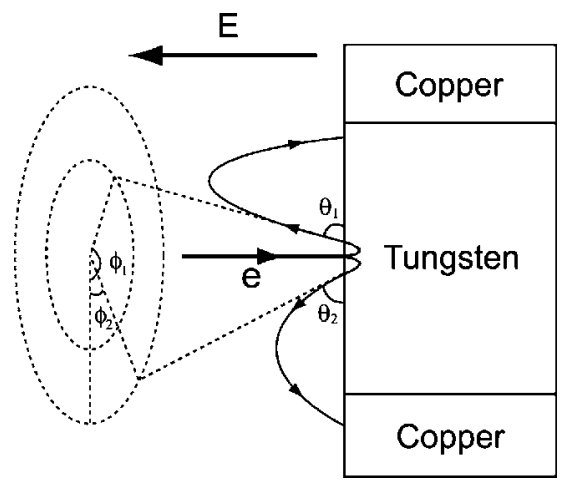

FIG. 1. Two electrons of an incoming pencil beam are backscattered with different backscattering angles $\left(\theta_{1}, \theta_{2}\right)$ and azimuthal angles $\left(\phi_{1}, \phi_{2}\right)$ with no external magnetic field.

fore the electrons can be characterized with only their backscattering energies and angle $(\theta)$. In order to obtain the combined energy and angular distribution of the secondary electrons, we studied the backscattering process with a Monte Carlo program, EGS4 (Electron Gamma Shower Ver. 4), which can simulate the coupled transport of electrons and photons in an arbitrary geometry for particles with energies from a few $\mathrm{keV}$ up to several $\mathrm{TeV}^{8}$

Although electrons can be backscattered for a second or even multiple times, the impact of higher order backscattered electrons should be small because these electrons are fewer in number, less likely to have sufficient energy, and more likely to land outside the target area. Here, we study electrons that are backscattered only once, which we term secondary electrons.

\section{Trajectories of secondary electrons}

The motion of the backscattered electrons, whose initial angular and energy distribution was determined using the EGS4 code, in the presence of combined electric and magnetic fields was modeled assuming both fields were uniform and aligned with the anode-cathode axis. The model ignored secondary effects such as the field distortion due to the focusing cup, the finite size of the anode, and the anode tilt angle. Under these assumptions, the electron motion has two

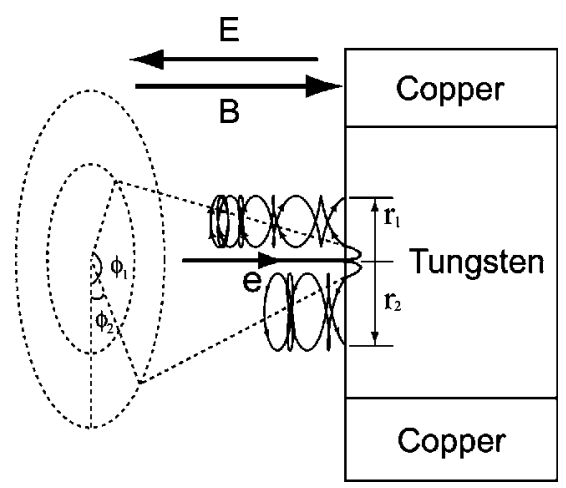

FIG. 2. Two electrons of an incoming pencil beam are backscattered in a magnetic field. 
components: a linear motion in the direction of the tube axis and a circular motion in the plane perpendicular to the tube axis. If the magnetic field is strong, the radius of the circular motion can be small and the backscattered electrons are more likely to return to locations close to the original backscattering sites (Fig. 2). Whereas primary electrons strike the target at an angle nearly normal to the anode surface, backscattered electrons have a wide distribution of incident directions. This factor was not considered in our calculation.

\section{Radiation output}

The x-ray spectrum and radiation exposure of an x-ray tube were predicted using a computer program SRS-78 (Refs. 9 and 10) based on work by Birch and Marshall ${ }^{11,12}$ which calculates $\mathrm{x}$-ray spectra in close agreement with experimental results. SRS-78 provides data including $\mathrm{x}$-ray spectra, air kerma (energy absorbed by air, kerma: kinetic energy released in matter) and first half value layers (HVLs) for electron energies ranging from $20 \mathrm{keV}$ to $150 \mathrm{keV}$ at $1 \mathrm{keV}$ intervals. We estimated filtration due to the glass envelope and $\mathrm{x}$-ray window of our tube to be that of 2.3-mm glass by matching the first HVL given by the program with the first HVL measured experimentally outside the magnetic field at several energies.

Since the exposure rate can be predicted with SRS-78, it can be easily compared with data measured with the ionization chamber. However, it is less straightforward to simulate focal spot images on the detector with the predicted $\mathrm{x}$-ray spectrum because processes to convert $\mathrm{x}$ rays to detector output are quite complicated. Based on the fact that a fairly large percentage $(>50 \%)$ of incoming $\mathrm{x}$ rays interact with the detector, ${ }^{13}$ we used the total energy of all incoming $\mathrm{x}$ rays to estimate the signal from the detector.

\section{B. Radiation exposure rate vs magnetic field strength}

Computer simulations were performed to predict the radiation exposure rate as a function of field strength, and simulation results were compared with experimental results.

\section{Computer simulation}

For estimating the total x-ray output we assumed that the focal spot is small compared to the target area. A pencil beam of $10^{6}$ electrons was modeled to hit the center of a round tungsten target (radius: $6 \mathrm{~mm}$ ) and the radiation exposure rate due to these primary electrons was predicted using SRS78. Secondary electrons emerged from locations very close to their incidence point and their energy and angular distribution was predicted with EGS4. Only secondary electrons with energies $\geqslant 30 \mathrm{keV}$ were traced in combined electric and magnetic fields as they moved away from and then back toward the anode. If the secondary electrons landed on the anode at distances less than the target radius, their contribution to the emitted radiation was calculated based on their energies. Simulations were performed at different x-ray tube voltages $(50,65$, and $80 \mathrm{kV})$ in magnetic fields ranging from 0 to $0.5 \mathrm{~T}$, the field at the imaging volume of our hybrid $\mathrm{X}$-ray/MR system.

\section{Experimental geometry}

A radiation dosimetry system (Model 9010 with a $60 \mathrm{~cm}^{3}$ ionization chamber, Radcal Corp., Monrovia, CA) was used. Since these systems are seldom used in high magnetic fields, we tested the consistency of the readout when the chamber was placed within the bore of the magnet and outside the magnet room. The dosimeter electronics were always outside the strong field. In the test, a $T_{c}-99 \mathrm{~m}$ source was used since its production of $140 \mathrm{keV}$ gamma rays should not be affected by the external magnetic field. The pancake chamber was first attached to the lead-shielded radiation source so that the source-to-chamber distance remained constant, and the cover of the lead cylinder could be removed and replaced. Inside the bore $(B \sim 0.5 \mathrm{~T})$, as shown in Fig. 3(d), the ionization chamber was positioned in two orientations, with its round plates parallel to $B$ (position 1) and perpendicular to $B$ (position 2). Compared to measurements outside the field, when the chamber was at $B \sim 0.5 \mathrm{~T}$ the readout was unchanged when $\mathbf{B}$ was perpendicular to the round plates [position 1 in Fig. 3(d)] and dropped by $\sim 3 \%$ when $\mathbf{B}$ was parallel to the round plates. This small change is likely due to deflection of the charge carriers when $\mathbf{B}$ is not parallel to the chamber electric field that is used to move the charge carriers in the direction perpendicular to the plates toward the electrodes. ${ }^{14}$ Nonetheless, since the maximum change of $3 \%$ was comparable to the measurement error, the effect of $\mathbf{B}$ on the ionization chamber was not considered further.

A wooden stand was built to hold our x-ray tube (BX-1, focal spot $0.5 \mathrm{~mm}^{2}$, anode angle $10^{\circ}$, Brand X-ray, Addison, IL) and measurements were taken at various field strengths by moving the stand to different locations relative to the MR scanner (Fig. 3). The ionization chamber was placed at the center of the X-ray field at a constant distance of $\sim 70 \mathrm{~cm}$ from the $\mathrm{x}$-ray focal spot. The $\mathrm{x}$-ray tube worked in a continuous fluoroscopic mode at $3 \mathrm{~mA}$ for all three kVp's (50, 65 , and 80). The baseline exposure measurement was performed outside the magnet room [Fig. 3(a)] where the residual magnetic field was $<5 \mathrm{G}(0.0005 \mathrm{~T})$. For measurements in fields lower than $0.15 \mathrm{~T}$, the stand was placed at one end of the scanner, where the fringe field of the MR magnet varied smoothly in the axial direction. The x-ray tube axis was on the magnet axis so that the magnetic field was parallel to the tube axis [Fig. 3(b)]. For fields between $0.15 \mathrm{~T}$ and $0.50 \mathrm{~T}$, the stand was located between the two "donuts" of the magnet [Fig. 3(c)]. At each location, the field at the focal spot was estimated as the average of the fields measured on both sides of the tube housing at the tube axis.

\section{Study of x-ray spectrum and HVL}

Since most secondary electrons have energies lower than primary electrons, the $\mathrm{x}$-ray spectrum is expected to change when radiation from secondary electrons is taken into account. The energy distribution of secondary electrons was 


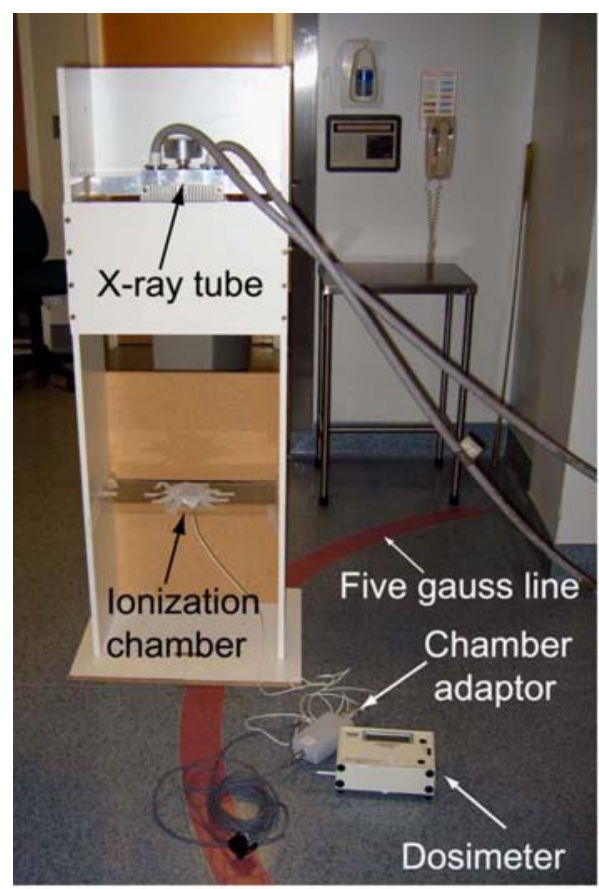

(a)

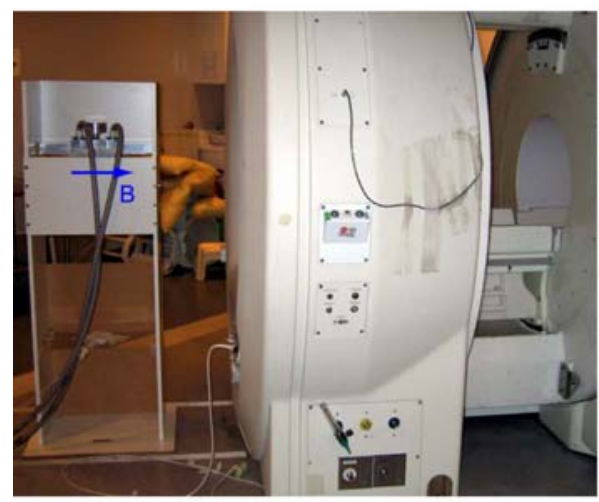

(b)

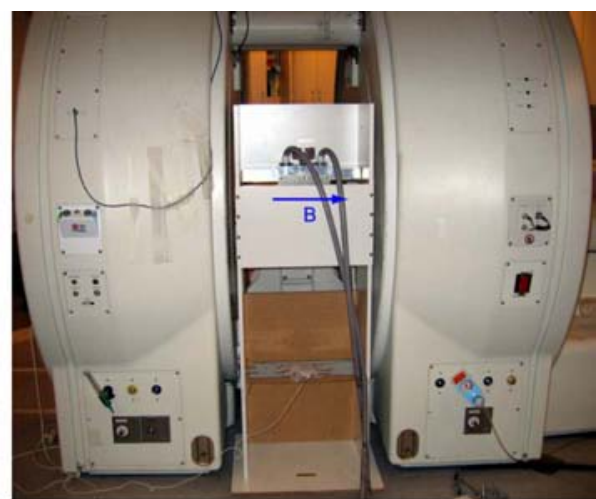

(c)

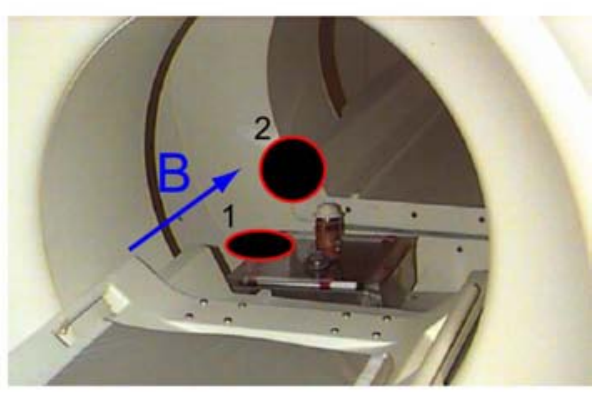

FIG. 3. The experimental setup for measurement of the $\mathrm{x}$-ray tube output in different magnetic fields: (a) outside the magnet room where the field is less than $0.0005 \mathrm{~T}$; (b) at one side of the scanner where the field is less than $0.15 \mathrm{~T}$; (c) in between the two "donuts" where the field can be as high as $0.5 \mathrm{~T}$; (d) ionization chamber in the magnet with parallel (1) and perpendicular (2) orientations. predicted with EGS4 and the spectrum of the X-ray emission was in turn calculated with SRS-78. The weighted sum of the $\mathrm{x}$-ray spectra from the secondary and primary electrons (weighted by the fraction of electrons at each energy) provided the estimated total $\mathrm{x}$-ray spectrum.

To characterize the energy distribution of the beam, we first calculated the first HVL using the simulated model by predicting the exposure rates with different thickness $\mathrm{Al}$ absorbers. We also measured the first HVL using an exponential fit to measurements with $0,0.5,1.0$, and $1.5 \mathrm{~mm} \mathrm{Al}$, with the tube placed at four different field strengths. The simulated HVL was compared with the measured HVL in a strong field $(0.5 \mathrm{~T})$ for which we assume that all the backscattered electrons hit the target area.

\section{Simulation of the spatial distribution of secondary radiation emission}

In order to calculate the distribution of secondary radiation emission from the anode, we first determined the point- spread function (PSF) of a pencil beam of electrons, i.e., the impulse response for the backscattering process as constrained by the magnetic field. The locations of impact of the secondary electrons on the anode were determined by their motion in parallel electric and magnetic fields as in Sec. II A 4. With the assumption that the secondary electrons are uniformly distributed over the $2 \pi$ azimuthal angle ( $\phi$ in Fig. 1 ), the radially symmetric electron distribution can be estimated as the spectrum of secondary electrons landing at an impact site as a function of the distance from the pencil beam. The PSF in terms of exposure or radiation intensity can be calculated from the relationship between exposure or intensity vs electron energy given by SRS-78. Convolution of this PSF and the primary electron density map on the anode then generates a map of secondary radiation emission. The sum of the secondary radiation and the primary radiation can then be projected in the direction of the observer (the ionization chamber or the detector). This calculated map of radiation intensity was compared with the focal spot image 


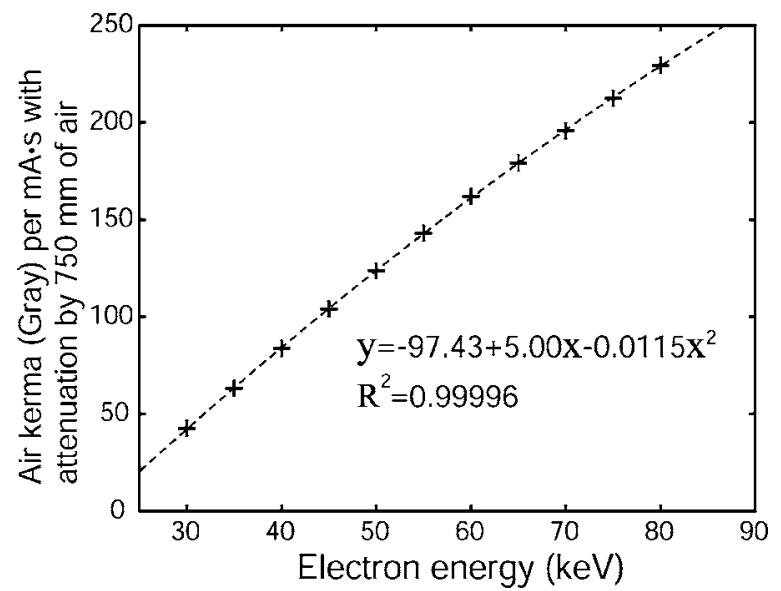

(a)

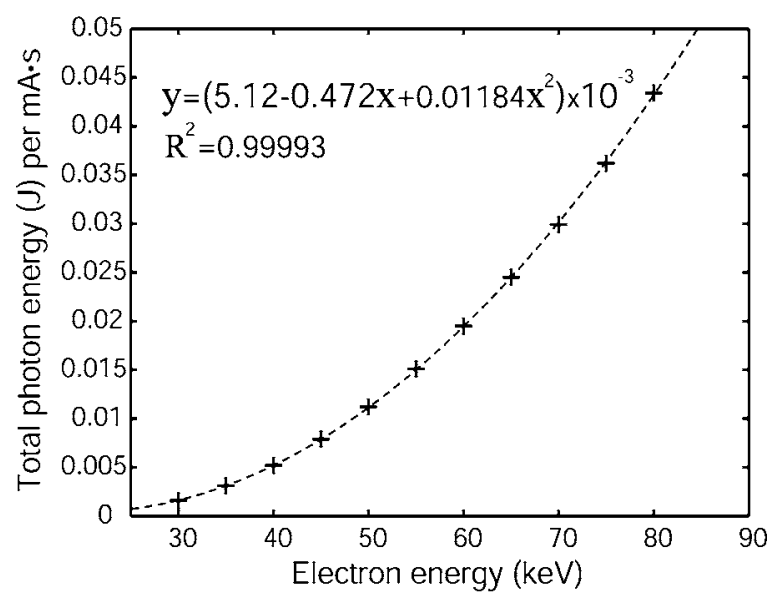

(b)

FIG. 4. Least-squares (LS) fitting was used to calibrate the relations between the amount of radiation and the electron energy based on 11 data points calculated with SRS-78: (a) radiation exposure rate vs electron energy; (b) total photon energy (x-ray intensity) vs electron energy.

collected using a $30 \mu \mathrm{m}$ pinhole (Pinhole assembly 07-613, Nuclear Associates, Long Island, NY) and our flat panel detector. Our setup had a magnification of $\sim 8.6$ for the focal spot (source to pinhole distance: $12.5 \mathrm{~cm}$, source to detector distance: $\sim 120 \mathrm{~cm}$ ). The $\mathrm{x}$-ray system worked in a continuous fluoroscopic mode at $3 \mathrm{~mA}, 65 \mathrm{kVp}$, and 30 frames per second. Fifteen to thirty frames of the focal spot images were averaged to improve image quality.

\section{RESULTS}

\section{A. Radiation exposure rate vs magnetic field strength \\ 1. Radiation exposure rate and intensity vs. electron energy}

Figure 4 shows that radiation exposure rate and total photon energy (proportional to $\mathrm{x}$-ray beam intensity) over a range of incoming electrons energies $(30-80 \mathrm{keV})$ can be approximated with second-order polynomial functions. Eleven data points with electron energies uniformly spaced

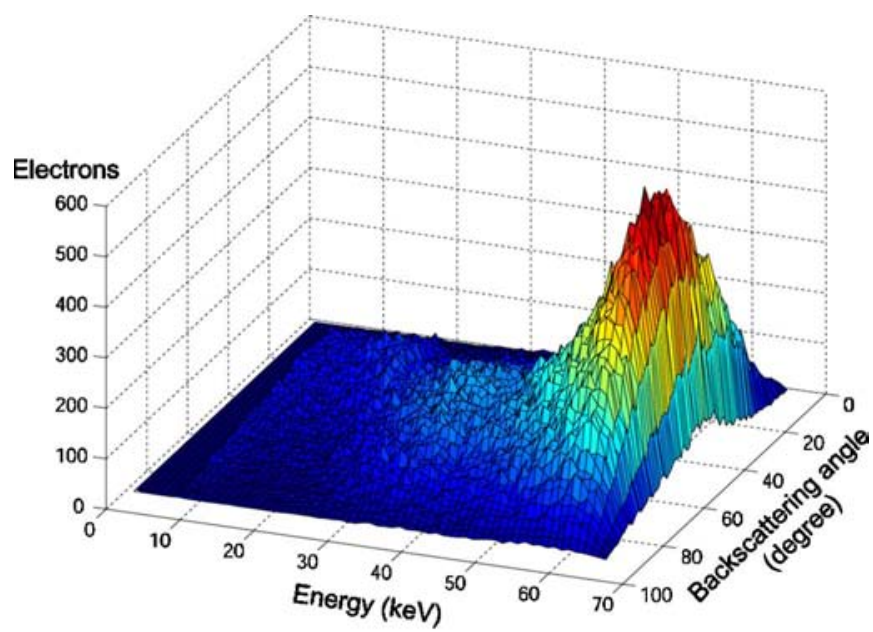

(a)
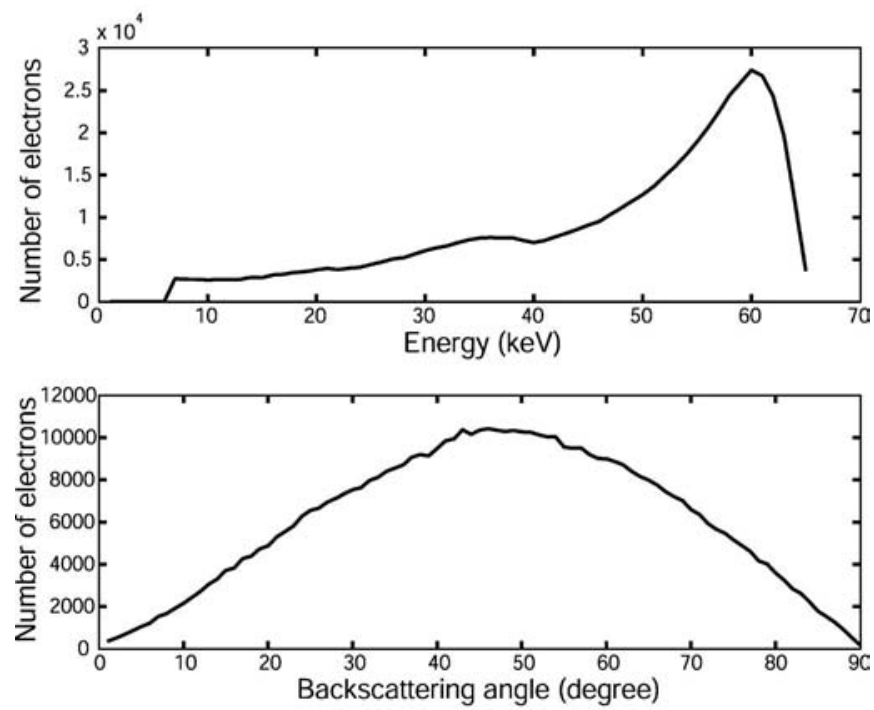

(b)

FIG. 5. The energy and angular distributions of secondary electrons simulated with EGS4 for $10^{6}$ primary electrons at $65 \mathrm{keV}$ : (a) combined energy and angular distribution; (b) separate energy and angular distributions.

in energy were calculated with SRS-78. The polynomial fits were used to calculate exposure and intensity at any electron energy.

\section{Energy and angular distribution of secondary electrons}

Figure 5 shows the EGS4 simulation results for $10^{6}$ primary electrons hitting a tungsten target with $65 \mathrm{keV}$. More than half $(54.4 \%)$ of the primary electrons were backscattered. Of the secondary electrons, $50.4 \%$ had energies higher than $80 \%$ of the energy of the primary electrons and $53.7 \%$ had backscattering angles between $30^{\circ}$ and $60^{\circ}$ [Fig. 5(b)].

\section{Radiation exposure vs $B$}

Figure 6 presents the predicted and measured radiation exposure rates vs field strength for three tube voltages (50, 65 , and $80 \mathrm{kV}$ ). As the magnetic field strength increases, the 


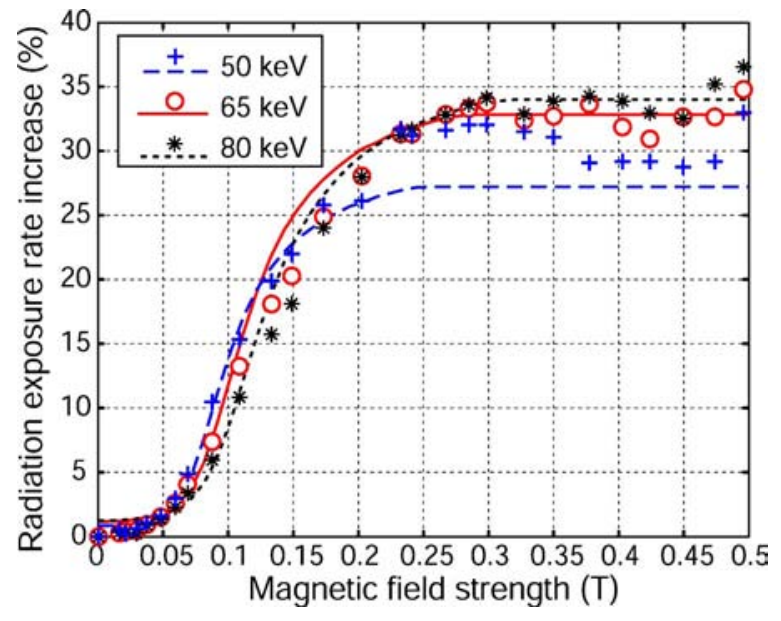

FIG. 6. Plot of radiation exposure rates vs magnetic field strengths at different tube voltages. Solid lines are simulation results and discrete points are experimentally measured data.

radii of rotations of the secondary electrons decrease, secondary electrons have increased probability of hitting the target a second time, and the x-ray output increases. Once the field is strong enough so that all of the electrons return to the target, the tube output is maximized and becomes independent of field strength. Good agreement between simulation and experimental results were found. At intermediate field strengths (around $0.1 \mathrm{~T}$ ), secondary electrons from the lowest energy primary electrons $(50 \mathrm{keV})$ resulted in the highest radiation increase since electrons with lower energies were easier to confine. At higher fields $(>0.3 \mathrm{~T})$, all the secondary electrons hit the target, and secondary electrons from the highest energy primary electrons $(80 \mathrm{keV})$ resulted in the largest increase $(\sim 34 \%)$. Discrepancies between the predicted radiation exposure and the measured data could result from a number of factors, which are examined in the Discus-

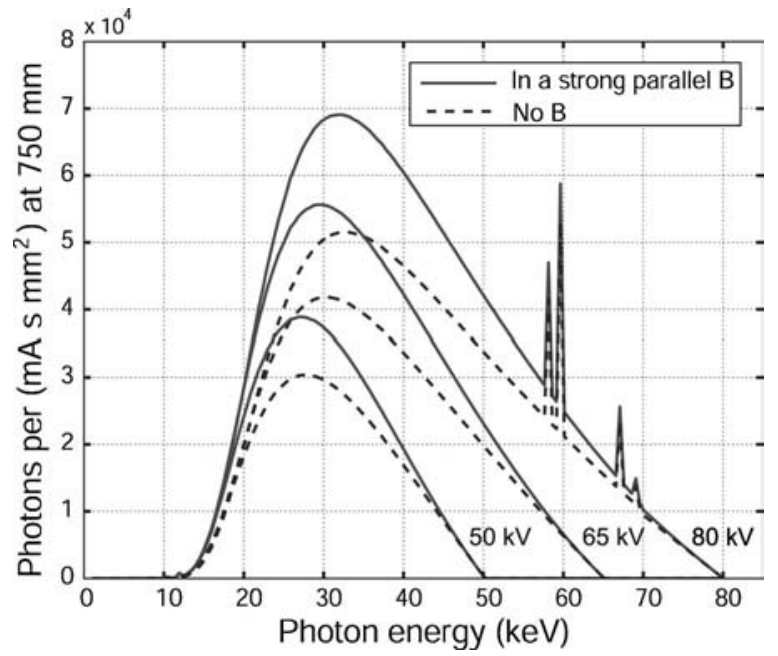

FIG. 7. Plot of x-ray spectra with and without a parallel strong magnetic field at 50, 65, and $80 \mathrm{kV}$.

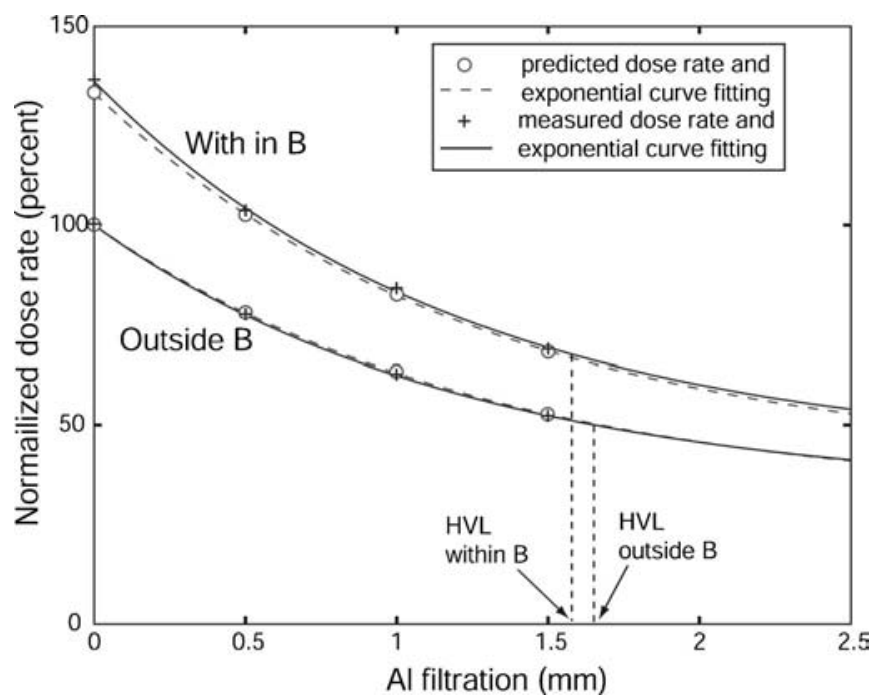

FIG. 8. Plot of relative $\mathrm{x}$-ray exposure rates as functions of Al filtration thickness within a strong magnetic field and outside a field with a tube voltage of $65 \mathrm{kV}$. Smooth lines were the fitting results of exponential functions.

sion. These results support the hypothesis that backscattered electrons are the cause of the observed increase in the tube output.

\section{B. X-ray spectra and HVL}

Figure 7 compares computed x-ray spectra with no magnetic field and in a strong magnetic field $(0.5 \mathrm{~T})$. With no magnetic field, very few secondary electrons land within the target area and the x-ray spectrum is essentially that of the primary electrons. In the strong field, all the backscattered electrons hit the target area with energies lower than that of the primary electrons and produce $\mathrm{x}$ rays with lower energies. As a result, the peak of the $\mathrm{x}$-ray spectrum in a strong magnetic field is slightly shifted towards lower energies.

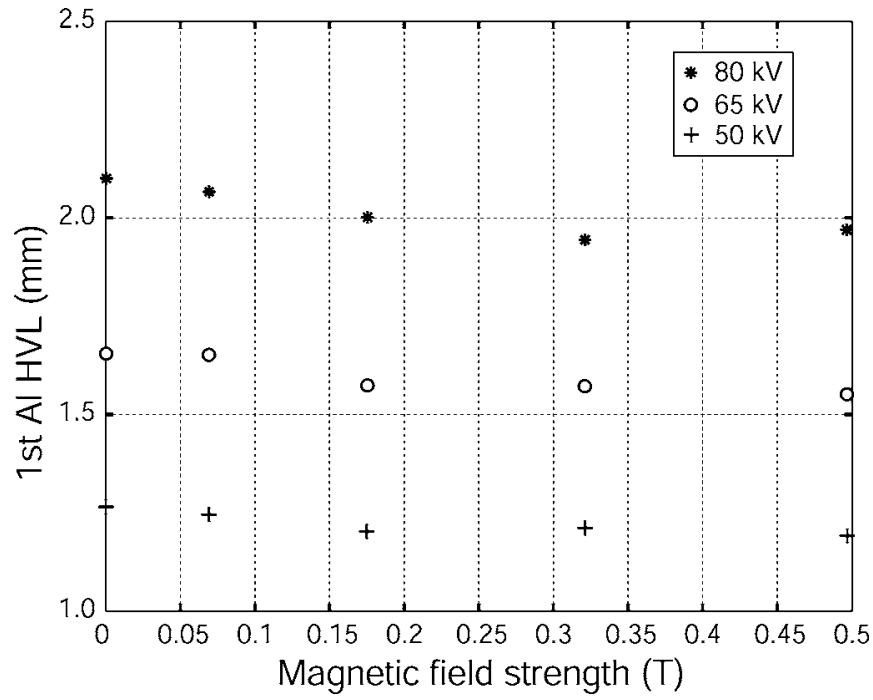

FIG. 9. Experimentally measured HVLs in different magnetic field strengths at three tube voltages $(50,65,80 \mathrm{kV})$. 
TABLE I. The first Al HVL with and without a strong field. Results were obtained from simulations and experiments at three tube voltages (50, 65, and $80 \mathrm{kV})$.

\begin{tabular}{cccccc}
\hline \hline \multirow{2}{*}{$\begin{array}{c}\text { Tube } \\
\text { voltage } \\
(\mathrm{kV})\end{array}$} & \multicolumn{2}{c}{ First Al HVL $(\mathrm{mm})$ with no magnetic field } & & \multicolumn{2}{c}{$\begin{array}{c}\text { First Al HVL (mm) with a } \\
\text { strong magnetic field }\end{array}$} \\
\cline { 2 - 3 } \cline { 5 - 6 } & SRS-78 & Simulation & Experiment & & Simulation \\
\hline 50 & 1.27 & 1.26 & 1.26 & $1.22(-3 \%)$ & $1.19(-6 \%)$ \\
65 & 1.67 & 1.67 & 1.66 & $1.58(-5 \%)$ & $1.55(-7 \%)$ \\
80 & 2.13 & 2.26 & 2.10 & $2.09(-8 \%)$ & $1.97(-6 \%)$ \\
\hline \hline
\end{tabular}

Excellent agreement was achieved between predicted and measured exposure rates with various $\mathrm{Al}$ filtrations for an $\mathrm{x}$-ray tube working at $65 \mathrm{kV}$ within and outside of a strong magnetic field (Fig. 8). As a result of the change of the X-ray spectrum, the HVL was reduced by around 6\% (Table I).

For all kV's, the HVL was reduced by a very small amount (less than 8\%) when the tube was operating in a strong magnetic field (Table I).

If the HVL is used as the criterion to evaluate the $\mathrm{x}$-ray spectrum, an x-ray tube working in a strong magnetic field generates a spectrum comparable to that of a tube working outside the field at a somewhat lower voltage. Specifically, $\mathrm{x}$-ray tubes working at 50,65 , and $80 \mathrm{kV}$ in a strong field are equivalent to tubes working at 48,62 , and $76 \mathrm{kV}$ outside the field. Alternatively, the spectrum of an x-ray beam can be characterized by the "effective energy" of a monochromatic $\mathrm{x}$-ray beam that has the same HVL as the polychromatic beam. Using this measure, the magnetic field causes a decrease of less that $1 \mathrm{keV}$ in the effective $\mathrm{x}$-ray energy at these applied voltages (Table II).

If the magnetic field is not strong enough to constrain all the backscattered electrons to return to the target, the intensity and HVL depend on the field strength. As shown in Fig. 9, for the $\mathrm{kVs}$ examined here, the HVLs decreased as the field strengthened until around $0.3 \mathrm{~T}$, above which the HVLs were no longer dependent on the field strength. Note that $0.3 \mathrm{~T}$ is also the field strength above which the intensity no longer increased with field strength (Fig. 6).

\section{Off-focal radiation}

\section{Secondary electron distribution of a pencil beam}

When the backscattered electrons return to the anode, they distribute around the landing point of the primary electron at different distances $(r)$ depending on their backscatter-

TABLE II. The energy of a monochromatic x-ray beam with the same HVL as the beam from an $\mathrm{x}$-ray tube with and within and without a strong field.

\begin{tabular}{ccc}
\hline \hline & \multicolumn{2}{c}{ Energy of equivalent monochromatic $\mathrm{x}$ rays $(\mathrm{keV})$} \\
\cline { 2 - 3 } $\begin{array}{c}\text { Tube voltage } \\
(\mathrm{kV})\end{array}$ & No magnetic field & In a strong field \\
\hline 50 & 24.2 & 23.9 \\
65 & 26.8 & 26.3 \\
80 & 30.0 & 29.1 \\
\hline
\end{tabular}

ing energies and angles (Fig. 2). At $65 \mathrm{kV}$ and with no magnetic field, only $10 \%$ of the backscattered electrons were within $6 \mathrm{~mm}$ from the center (Fig. 10). At a field of $0.2 \mathrm{~T}$, more than $90 \%$ of the secondary electrons were within a radius of $6 \mathrm{~mm}$. At $0.5 \mathrm{~T}$, almost all of the secondary electrons returned within a radius of $3 \mathrm{~mm}$.

The two dimensional PSF of secondary electron density at $65 \mathrm{kV}$ and $0.5 \mathrm{~T}$ is shown in Fig. 11(a). The dependence of the PSF on $B$ is illustrated in Fig. 11(b), where the PSF is plotted in a logarithmic scale at $B=0,0.2$, and $0.5 \mathrm{~T}$. The PSF with no field is spread over a large area compared to the PSF with a strong field. The oscillation of the PSF close to the origin is probably an artifact and could result from placing outgoing secondary electrons in bins with $1 \mathrm{keV}$ and $1^{\circ}$ increments. The effect of the discreteness of the energy and angular distribution of the electrons can be appreciated when there is no magnetic field and the secondary electrons spread broadly back on the target.

A qualitative comparison between simulated and experimental focal spot images is shown in Fig. 12. The window and level for each set of images were chosen such that the off-focal radiation could be appreciated (i.e., the gray scale within the spot is saturated). The off-focal radiation appears elliptical because the dimension along the cathode-anode axis was compressed when the focal spot image was pro-

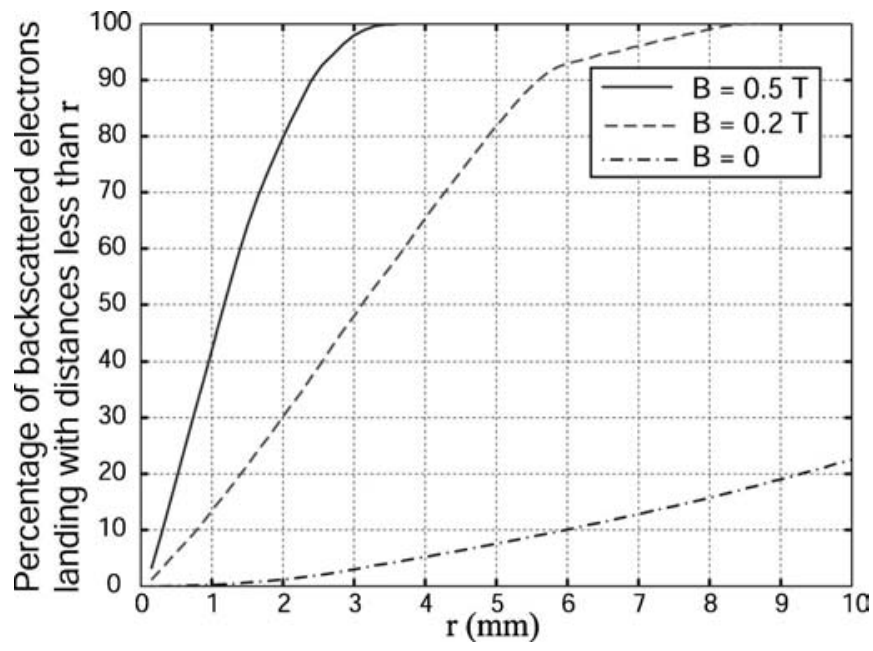

FIG. 10. Percentage of secondary electrons that return to the anode within the distance of $\mathrm{r}$ from the pencil beam of primary electrons in an $\mathrm{x}$-ray tube working at $65 \mathrm{kV}$. 

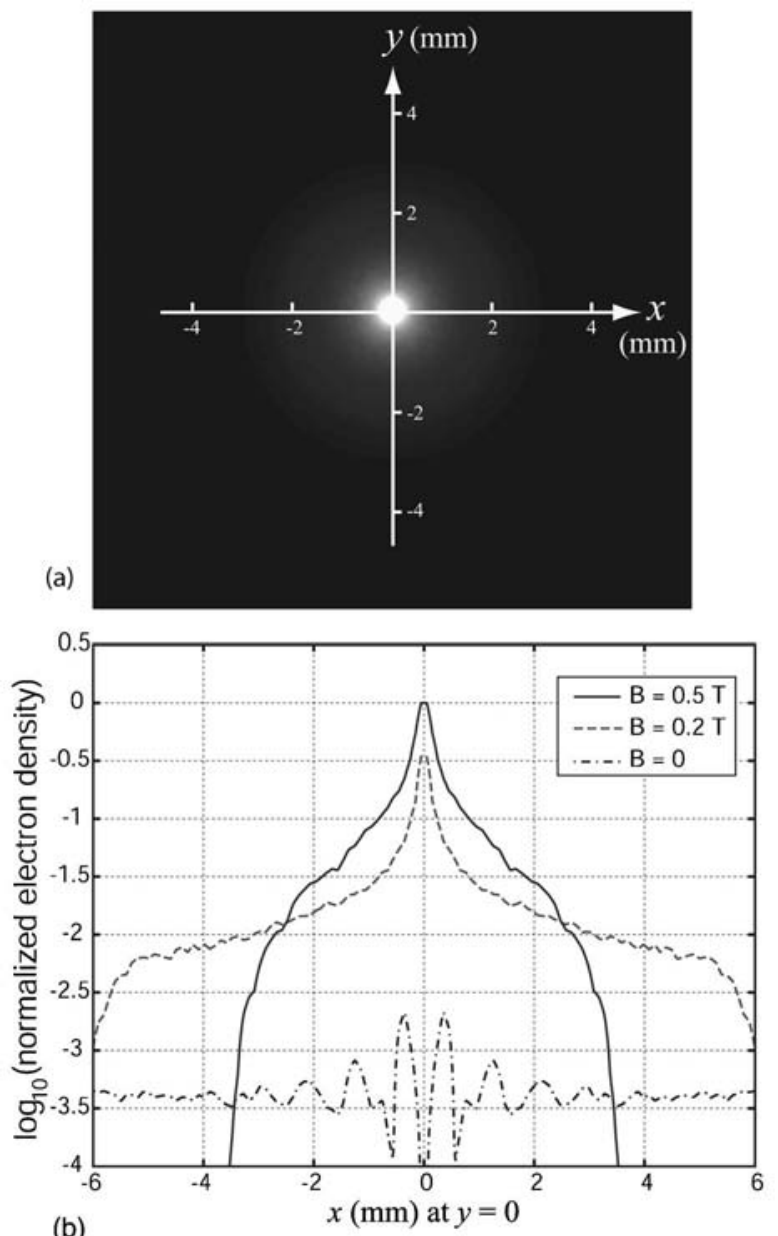

(b)

FIG. 11. (a) PSF of secondary electron density for a tube working at $65 \mathrm{kV}$ and in a field of $0.5 \mathrm{~T}$; (b) the PSF on the $x$ axis in a logarithmic scale with $\mathrm{B}=0,0.2$ and $0.5 \mathrm{~T}$.

jected in the direction of the detector. As the magnetic field strength increases, the off-focal radiation becomes more localized and more intense.

Along the vertical direction of the focal spot images in Fig. 12, the off-focal radiation appears as decreasing tails on both sides of the focal spot. The ratio of the peak of the focal spot to the tail is largest at the edge of the focal spot. Figure 13 shows the profiles of the focal spot along the tails of off-focal radiation for an $\mathrm{x}$-ray tube working at $65 \mathrm{kV}$ and in a field of $0.5 \mathrm{~T}$ with arrows indicating edges of the focal spot. For the focal spot calculated using the computer simulation, the peak-to-tail ratio was $10^{1.6}(\approx 40)$, while the ratio observed in the experiment was $10^{1.2}(\approx 16)$.

Theoretically the increased output of an x-ray tube working in a strong magnetic field due to secondary radiation could originate from anywhere within the target area, but only the radiation originating within or near the focal spot can be beneficial to the x-ray image, while the diffuse radiation (off-focal radiation) may reduce image quality. Using our finite element model, secondary radiation exposure within the focal spot area was plotted as a function of field strength [Fig. 14(a)]. At 0.2 T, although the magnetic field
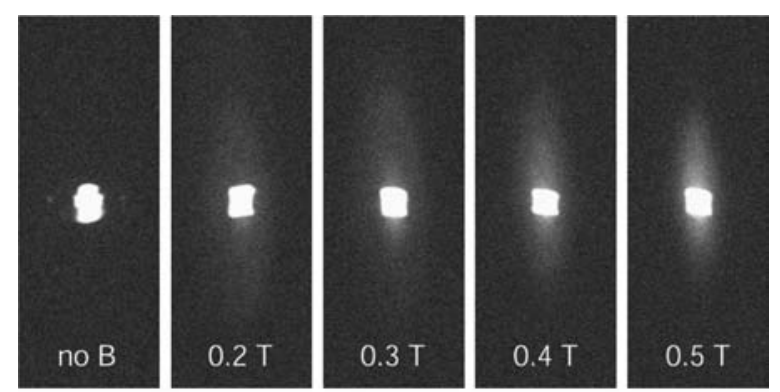

(a)
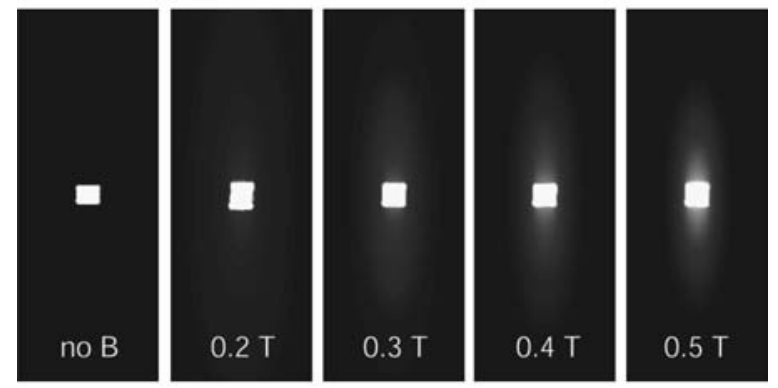

(b)

FIG. 12. Focal spot images at magnetic field strengths ranging from 0 to $0.5 \mathrm{~T}$ : (a) computer simulated images; (b) focal spot images obtained with an x-ray detector.

constrained nearly all the secondary electrons within the target area, less than $10 \%$ of the secondary radiation originated from the focal spot. It was not until $1.3 \mathrm{~T}$ that half of the secondary radiation was produced in the focal spot. The secondary radiation emanating from a $1 \times 1 \mathrm{~mm}^{2}$ area centered on the projected focal spot was also computed [Fig. 15(b)]. A field of $0.7 \mathrm{~T}$ was required to confine half of the secondary radiation inside the box. In Fig. 15(b), the length of the focal spot is larger than its nominal size of $0.5 \mathrm{~mm}$ because the magnetic field is so strong that it overpowers the electrostatic focusing effect of the x-ray tube.

Since the system modulation transfer function (MTF) depends on the geometry of the focal spot, ${ }^{15}$ the MTF can be

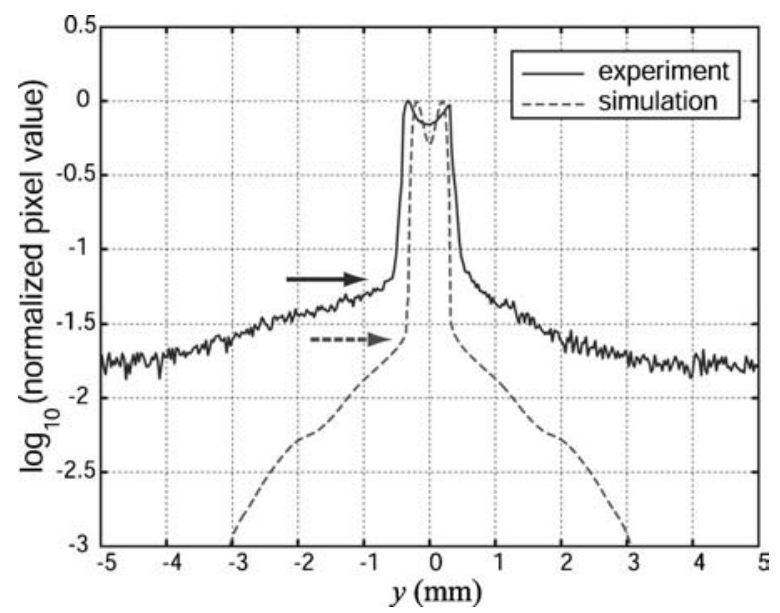

FIG. 13. Profiles of the focal spot of an $\mathrm{x}$-ray tube working at $65 \mathrm{kV}$ and in a field of $0.5 \mathrm{~T}$ (arrows indicate the edges of the focal spot). 


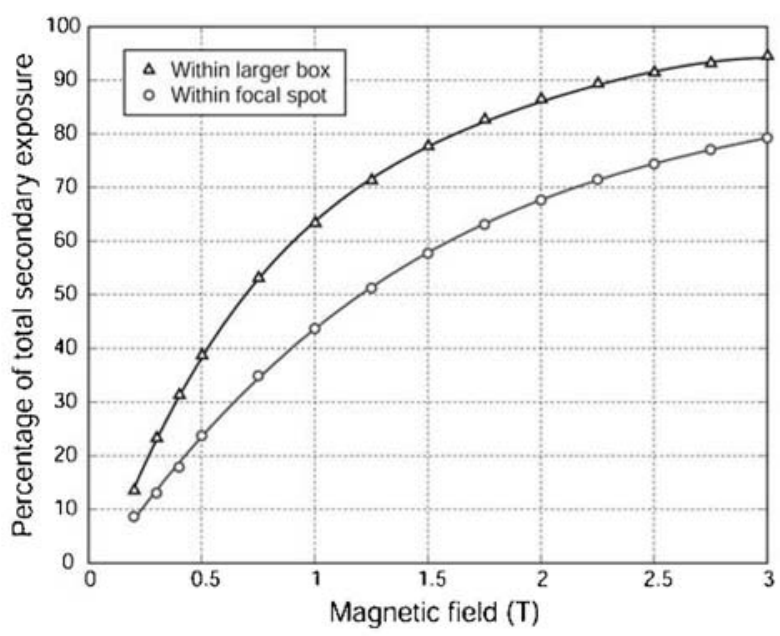

(a)

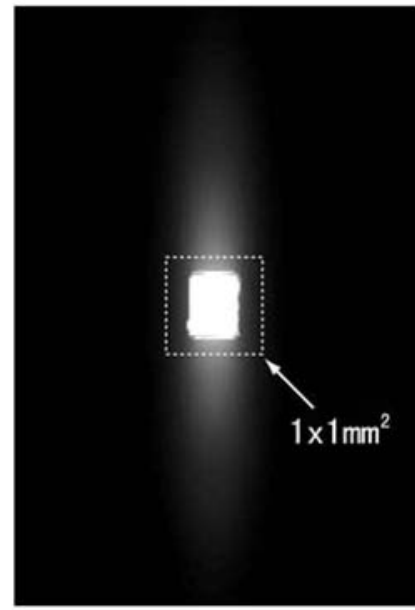

(b)

FIG. 14. (a) Plot of the percentage of secondary radiation within the focal spot area and within a larger box area as functions of the magnetic field strength (tube voltage: $65 \mathrm{kV}$ ); (b) the $1 \times 1 \mathrm{~mm}^{2}$ box area centered around the focal spot at $0.5 \mathrm{~T}$.

degraded by increased off-focal radiation when the $\mathrm{x}$-ray tube is used in a magnetic field. This degradation is difficult to measure experimentally, but can be estimated with simulation data. The contribution of the focal spot to the system MTF is its Fourier transform, scaled by the magnification of the focal spot on the detector. Due to the anode tilt, changes in this direction are diminished by the anode angle and should be insignificant; therefore, integration was performed along this direction [horizontal direction in Fig. 14(b)]. The MTF was then obtained by calculating the one-dimensional Fourier transform in the perpendicular direction [vertical direction in Fig. 14(b)]. Results are shown in Fig. 15 for a system in different magnetic fields with equal SOD (sourceto-object distance) and ODD (object-to-detector distance), i.e., in an image with a magnification of one for the focal spot and magnification of two for the object.

Figure 15(a) shows the MTF without considering secondary radiation. The difference among the MTFs is due to the fact that the magnetic field affected the electron trajectories, leading to different primary electron distributions. ${ }^{5}$ With no magnetic field, the MTF was the highest because the electrostatic focusing was not disturbed and the focal spot size was minimized [Fig. 12(a)]. At $0.2 \mathrm{~T}$, the focal spot was wider than those at other fields, and therefore the corresponding MTF was minimized. For $B \geqslant 0.3 \mathrm{~T}$, the focal spot sizes were similar and so were the MTFs.

Figure 15(b) shows the MTFs with the effect of secondary radiation. For $B \leqslant 0.5 \mathrm{~T}$, most of the secondary radiation was off-focal and widely distributed. As a result, the effect on the MTF is most evident as a sharp drop at low spatial frequencies. Similar effects were observed experimentally. ${ }^{3}$

\section{DISCUSSION}

A number of assumptions and simplifications were made in simulations of the physical processes that occur in the $\mathrm{x}$-ray tube. In predicting the tube output as a funcition of $B$, the electron beam was assumed to be an infinitesimally thin pencil beam, the anode surface was assumed to be perpendicular to the tube axis, and only secondary electrons were considered. More sophisticated models could account for the finite size of the beam, the distortion of electric field due to the tilted anode, and multiply backscattered electrons at all energies. In addition, only electrons with energies higher than $30 \mathrm{keV}$ were considered. This assumption, which would have the largest impact on the lowest tube voltage, likely led to underestimation of the radiation increase at $50 \mathrm{kV}$. Some of the discrepancies between the experimental data points and the predicted curves in Fig. 6 can also be attributed to the unavoidable measurement errors in the magnetic field strength and exposure rate, and the stability of the x-ray system and the dosimeter system.

To predict pixel values on the detector, the detector was assumed to have $100 \%$ sensitivity to $\mathrm{x}$-ray photons and the signal was assumed to be linearly dependent on the total energy. In addition, dark current and electronic noise were not taken into consideration. Imperfect dark current subtraction and noise are the probable cause of the background under the off-focal radation, leading to the lower apparent peak-to-tail ratio shown in Fig. 13.

The secondary radiation is difficult to image since it is more spatially distributed and has low magnitude. Since the magnetic field changes the size and shape of the focal spot, it is challeging to obtain an image of the focal spot inside a magnetic field without secondary radiation. Therefore, it is not easy to experimentally separate the secondary radiation from the primary radiation in the focal spot area. At the same time, due to its low signal level, the secondary radiation may not be accurately measured outside the focal spot in the presence of background noise and possibly variable dark current. Hence, the distribution map of secondary radiation based on calculations is especially helpful in evaluating the increased radiation. 


\section{focal spot without secondary radiation}

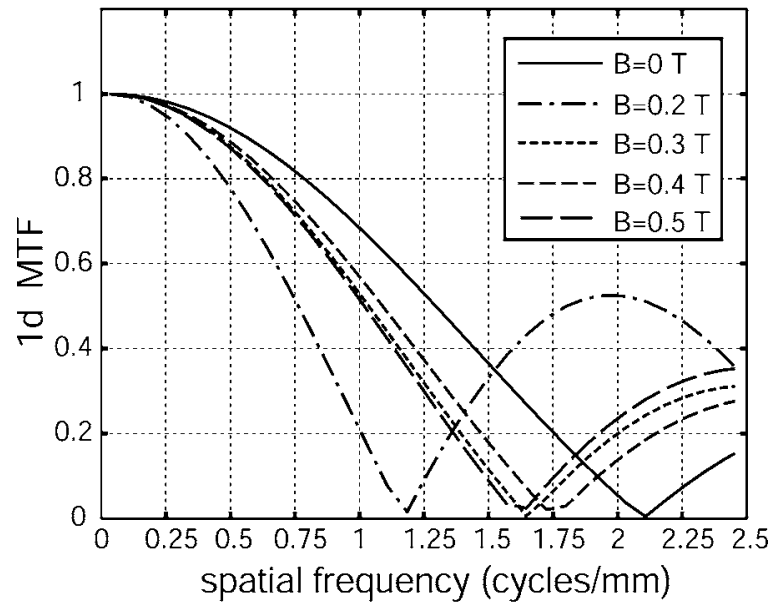

(a)
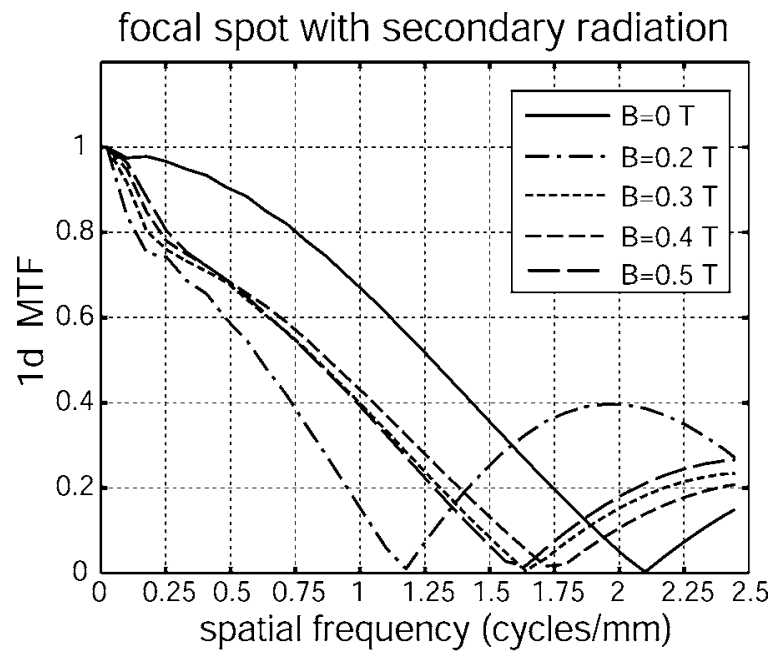

(b)

FIG. 15. One-dimensional (1d) MTF due to the finite focal spot size in magnetic fields ranging from 0 to $0.5 \mathrm{~T}$ based on simulation data: (a) without secondary radiation; (b) with secondary radiation.

The off-focal radiation, if not confined near the focal spot area, can degrade the MTF. This effect may partially account for the change in MTF previosuly measured at the patient position when the $\mathrm{x}$-ray source was placed in a magnetic field of $0.2 \mathrm{~T}^{3}$. Since the magnetic field also affects the primary electrons, ${ }^{5}$ careful analysis is needed to differentiate the impacts on MTF from these two factors.

The increase in tube output may not be as strong when a rotating anode $\mathrm{x}$-ray tube is placed in a strong magnetic field. Rotating anode tubes have much larger targets. It is likely that most of the backscattered electrons return to the anode and produce off-focal $\mathrm{x}$ rays even without a magnetic field. Introduction of a magnetic field to the tube may change the distribution of its focal and off-focal radiation, but may not greatly increase the total tube output.

\section{CONCLUSIONS}

When an x-ray tube is placed in a magnetic field that is parallel to the tube axis, the tube output increases with in- creasing field strength due to the effect of backscattered electrons. More than half of $65 \mathrm{keV}$ primary electrons are backscattered when they strike the anode. Without a magnetic field, only a small fraction of these electrons return to the target of a fixed anode tube, and they produce primarily offfocal radiation. If the tube is placed in a magnetic field parallel to the tube axis, the backscattered electrons spiral around the magnetic field lines and have increased probability of hitting the target again. Therefore, the x-ray tube output increases with increasing magnetic field strength. The observed total exposure, HVL, and focal spot image, which agree well with computer simulations, can also predict quantities that may be difficult to measure, such as the x-ray spectrum and the distribution of secondary radiation on the anode. The $\mathrm{x}$-ray spectrum is shifted slightly towards lower energies and the half value layer is reduced by about $6 \%$. In a magnetic field of $0.5 \mathrm{~T}$, all backscattered electrons are confined in the target area of our x-ray tube. As a result, the exposure is increased by $33 \%$. However, only a small fraction of the additional radiation is produced within the focal spot area and the system MTF at low spatial frequencies is degraded. To confine at least half of the secondary emission to the focal spot, a field of $\sim 1.2 \mathrm{~T}$ is needed.

A magnetic field can be used to increase the $\mathrm{x}$-ray tube output by confining the backscattered electron within the tungsten area on the anode. The spatial distribution of secondary radiation can be controlled by the field strength. The size of the tungsten area determines the minimum field strength needed to reach the maximum tube output.

\section{ACKNOWLEDGMENTS}

This work was supported by NIH Grants Nos. RR09784 and EB000198, GE Healthcare, and the Lucas Foundation.

${ }^{1}$ R. Fahrig et al., "A truly hybrid interventional MR/x-ray system: Feasibility demonstration," J. Magn. Reson Imaging 13, 294-300 (2001).

${ }^{2}$ R. Fahrig et al., "Truly hybrid interventional MR/x-ray system: Investigation of in vivo applications," Acad. Radiol. 8, 1200-1207 (2001).

${ }^{3}$ R. Fahrig et al., "Performance of a static-anode/flat-panel x-ray fluoroscopy system in a diagnostic strength magnetic field: A truly hybrid X-ray/MR imaging system," Med. Phys. 32, 1775-1784 (2005).

${ }^{4}$ G. V. Pavlinsky and A. Y. Portnoy, "Formation features of radiation from x-ray tubes with grounded cathode," X-Ray Spectrom. 31, 247-251 (2002).

${ }^{5}$ Z. Wen, R. Fahrig, and N. J. Pelc, "X-ray tube in parallel magnetic fields," in Proceedings of the SPIE Medical Imaging Meeting, 2003, Vol. 5030, pp. 972-979.

${ }^{6} \mathrm{Z}$. Wen, R. Fahrig, S. Conolly, and N. J. Pelc, "Investigation of electron trajectories of an X-ray tube in magnetic fields of MR scanners" (submitted).

${ }^{7}$ Z. Wen, R. Fahrig, and N. J. Pelc, "Robust x-ray tubes for use within the magnetic field of an MR scanner," Med. Phys. 32, 2327-2336 (2005).

${ }^{8}$ W. R. Nelson, H. Hirayama, and D. W. O. Rogers, The EGS4 Code System. SLAC-265 (1985).

${ }^{9}$ K. Cranley, B. Gilmore, G. Fogarty, and L. Desponds, Catalog of diagnostic x-ray spectra and other data. The Institute of Physics and Engineering in Medicine (www.ipem.ac.uk) Report No. 78 (1997).

${ }^{10} \mathrm{P}$. Meyer et al., "Evaluation of the use of six diagnostic x-ray spectra computer codes," Br. J. Radiol. 77, 224-230 (2004).

${ }^{11} \mathrm{R}$. Birch and M. Marshall, "Computation of bremsstrahlung x-ray spectra and comparison with spectra measured with a $\mathrm{Ge}(\mathrm{Li})$ detector," Phys. Med. Biol. 24, 505-517 (1979). 
${ }^{12}$ R. Birch, M. Marshall, and G. M. Ardran, 1979 Catalog of Spectral Data for Diagnostic X-Rays Hospital Physicists' Association Scientific Report Series 30 (IPSM, P.O. Box 303, York, UK).

${ }^{13}$ J. H. Siewerdsen et al., "Empirical and theoretical investigation of the noise performance of indirect detection, active matrix flat-panel imagers
(AMFPIs) for diagnostic radiology," Med. Phys. 24, 71-89 (1997).

${ }^{14} \mathrm{~J}$. T. Bushberg, The Essential Physics of Medical Imaging (Lippincott Williams and Wilkins, Philadelphia, 2002).

${ }^{15} \mathrm{H}$. E. Johns and J. R. Cunningham, The Physics of Radiology (Charles C. Thomas, Springfield, 1983). 